\title{
Treating Constraints as Objectives in Multiobjective Optimization Problems Using Niched Pareto Genetic Algorithm
}

\author{
Douglas A. G. Vieira, Ricardo L. S. Adriano, João A. Vasconcelos, and Laurent Krähenbühl
}

\begin{abstract}
In this paper, the constraints, in multiobjective optimization problems, are treated as objectives. The constraints are transformed in two new objectives: one is based on a penalty function and the other is made equal to the number of violated constraints. To ensure the convergence to a feasible Pareto optimal front, the constrained individuals are eliminated during the elitist process. The treatment of infeasible individuals required some relevant modifications in the standard Parks and Miller elitist technique. Analytical and electromagnetic problems are presented and the results suggest the effectiveness of the proposed approach.
\end{abstract}

Index Terms-Constrained problems, elitism, multiobjective optimization.

\section{INTRODUCTION}

W HEN genetic-based algorithms are used, the most common way of handling constraints is by using penalty techniques like the exterior penalty method. There are some weaknesses in this classical approach because good values for the penalty parameters are not known.

In this paper, constraints in constrained multiobjective optimization problems (CMOPs) are handled as objectives and the niched pareto genetic algorithm (NPGA) [1] solves the resulting problem. Although all the real constraints could be added to the objectives, this can be problematic in case when the number of constraints is very high, adding too much objectives to the optimization process, making the search much more complex [2]. To avoid this problem just two functions are added to the objectives independently of the number of constraints. These are a penalty function, which can be viewed as a measure of the distance between the feasible and infeasible regions, and the number of violated constraints.

The original NPGA was modified by incorporating the Parks and Miller elitist technique (P\&M) [3], which needed some changes when constraints were treated as objectives. The required changes were essential to avoid convergence toward an infeasible space.

Analytical test problems, which were designed with special features to difficult the Pareto-Optimum (PO) front search, are chosen to compare both approaches of treating constraints: 1) as objectives

Manuscript received July 1, 2003. This work was supported in part by CNPq under Grant 350.902/1995 - 0 and in part by CAPES/COFECUB under Grant 318/00-11.

D. A. G. Vieira, R. L. S. Adriano and J. A. Vasconcelos are with the Electrical Engineering Department, Federal University of Minas Gerais, 31270-901 Belo Horizonte, Minas Gerais, Brazil (e-mail: douglas@cpdee.ufmg.br; rluiz@ cpdee.ufmg.br; joao@cpdee.ufmg.br).

L. Krähenbühl is with CEGELY-ECL, 69131 Ecully Cedex, Lyon, France (e-mail: Laurent.Krahenbuhl@ec-lyon.fr).

Digital Object Identifier 10.1109/TMAG.2004.825006 and 2) using penalty function to tranform the original constrained problem into an unconstrained one. Two quantitative metrics were adopted here to compare the results from both approaches: generational distance (GD), which is a measure of the distance between the desired and found fronts, and the number of solutions found by the algorithm [4]. This choice enabled a realistic sight of the techniques discussed. After that, both classical and new procedures were applied to find the nondominated front by solving a CMOP based on the TEAM22 problem [6].

\section{MATHEMATICAL FORMULATION}

The multiobjective optimization involves $m$ objective functions, $n$ constraints and a set of $k$ decision variables. In terms of minimization we can write this problem as

$$
\begin{aligned}
& \operatorname{minimize} \\
& \qquad \bar{f}=\left\{f_{1}(\bar{x}), f_{2}(\bar{x}) \cdots f_{m}(\bar{x})\right\}^{T} \\
& \text { subjected to } \\
& \quad \bar{g}=\left\{g_{1}(\bar{x}), g_{2}(\bar{x}) \cdots g_{n}(\bar{x})\right\}^{T} \leq\{0,0 \cdots 0\}^{T}
\end{aligned}
$$

where $\bar{x}=\left\{x_{1}, x_{2} \cdots x_{k}\right\}^{T}$.

As NPGA is not capable to deal directly with constrained problems, some way must be found to handle the constraints. In this paper, two approaches are considered. First, the constraints are incorporated to the fitness function by using penalty functions. This procedure will be denoted here as classical approach. Initially, the original optimization problem is rewritten as an unconstrained one. For instance, for the $i$ th objective, a pseudo-objective function $f f_{i}$ can be written as

$$
f f_{i}(\bar{x})=f_{i}(\bar{x})+\sum_{j=1}^{n} \rho_{j}\left(g_{j}(\bar{x})\right)_{+}^{2}
$$

where $\rho_{j}$ is the $j$ th penalty parameter associated to the $j$ th constraint and ()$_{+}$denotes that only violated constraints are considered. Usually, all $n$ penalty parameters are taken with the same value, i.e., $\rho_{j}=\rho$ for $j=1, \ldots, n$.

Second, the $n$ constraints are transformed in two more objectives, the penalty function and the number of violated constraints, say w. To avoid confusion, this approach will be denoted here as novel approach. Mathematically, the original problem is rewritten as

$$
\begin{aligned}
& \operatorname{minimize} \\
& \bar{f}=\left\{f_{1}(\bar{x}) \cdots f_{m}(\bar{x}), \sum_{j=1}^{n} \rho_{j}\left(g_{j}(\bar{x})\right)_{+}^{2}, w\right\}^{T} .
\end{aligned}
$$

Using both approaches the constrained problem is transformed into an unconstrained one, which can be solved using some multiobjetive genetic-based algorithm. 


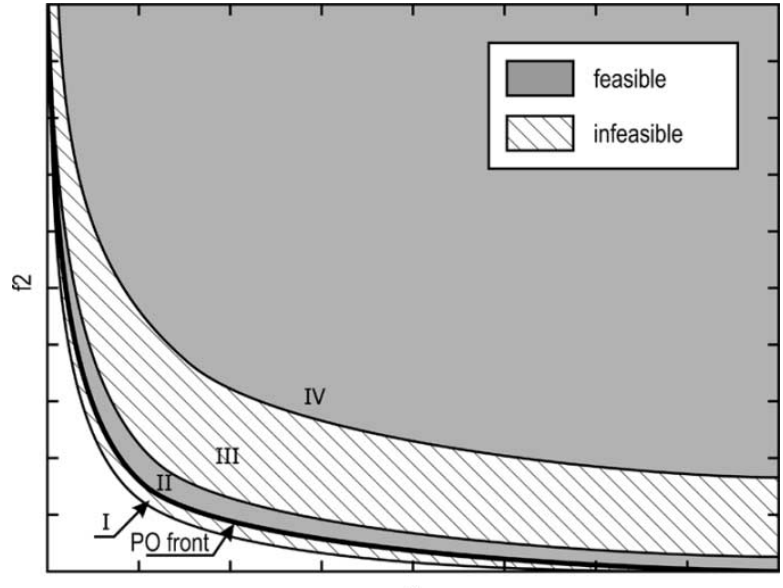

f1

Fig. 1. Hypothetical problem.

\section{TREATING CONSTRAINTS AS OBJECTIVES}

Why is treating the constraints as objectives interesting? For instance, imagine a hypothetical problem, in terms of multiobjective minimization, as illustrated in Fig. 1.

The regions II and IV are feasible ones but I and III are infeasible. As can be seen region IV is the biggest one and probably more samples will be generated in this region in the first population. Using the classical approach the individuals of region III will be dominated by those of region IV, because all objectives evaluated on points belonging to region III will be increased by a penalty value, guiding the search in direction of region IV. Region III is as a "wall" between regions II and IV. Suppose that in the first population no point is sampled in region II, which is the most probable situation because this is the shortest region and it is very small. So finding a point belonging to this region would be a hard task. Therefore, one expects that the algorithm will converge most of times to the front defined in the region IV. On the other hand, when handling constraints as objectives III is not viewed as a "wall" because points of region IV do not dominate those of III.

Considering the analysis done previously, it is clear that the approach of handling constraints as objectives can be very effective. However, some problems can occur when the region I is considered. One constrained PO front is defined in region I, and the algorithm can be guided to find this front despite of the front defined in region II. As the front of interest is defined in region II, this situation must be avoided. Due to these problems some modifications were required in the elitist procedure, and it is described in the Section IV.

\section{MODIFIED P \& M ELITISM}

As is well known, the performance of genetic-based algorithms can be improved using elitist procedures. In this paper, the Parks \& Miller elitist technique [3] was used. It consists in incorporating the efficient individuals of the on-line population $\left(\mathrm{P}_{\text {on }}\right)$ to the off-line population $\left(\mathrm{P}_{\text {off }}\right)$, at each generation. When $\mathrm{P}_{\text {off }}$ size exceeds a threshold, the dominance criterion is applied, eliminating all dominated solutions. If $\mathrm{P}_{\text {off }}$ size continues bigger than the threshold, a distance criterion is applied. It is based on measuring the distance between the off-line individuals, taking two per turn, and if they are

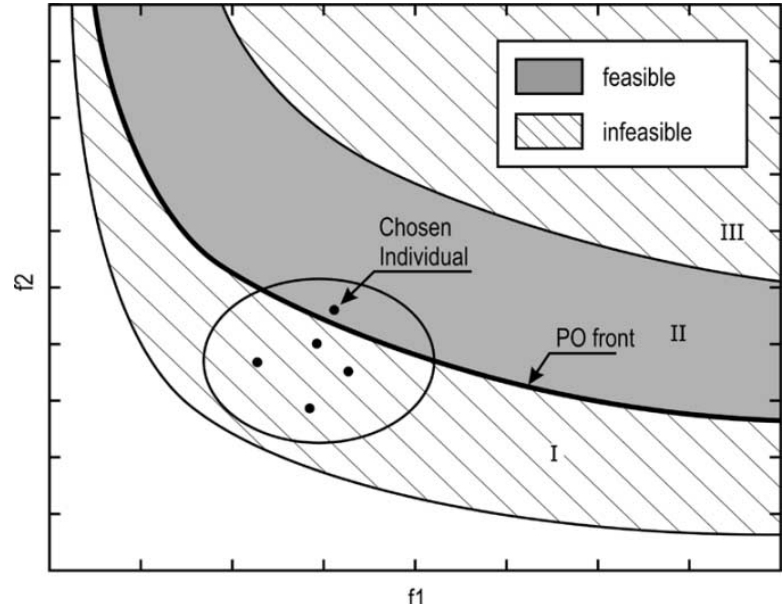

Fig. 2. Eliminating infeasible individuals.

within some distance one of them is discarded, chosen randomly. This distance is measured in the objective space. The individuals of $\mathrm{P}_{\text {off }}$ are often reinserted in $\mathrm{P}_{\text {on }}$ to improve the convergence. This approach works well when the constraints are considered in the classical approach.

Even though infeasible individuals (infeasible points) do not represent the wished PO front, keeping them in the on-line population seems to be a good idea because infeasible points close the PO front might be lost during the optimization process. When using constraints as objectives, i.e., the new approach, P\&M elitism does not work properly because sometimes the $\mathrm{P}_{\text {off }}$ may be composed by a great deal of infeasible points, guiding the search to an wrong direction. To avoid this drawback, P\&M technique was modified by two additional procedures to avoid convergence to infeasible regions: 1) when eliminating individuals of $\mathrm{P}_{\text {off }}$ by the distance criterion, infeasible points are discarded if they are near to a feasible one and 2) when $\mathrm{P}_{\text {off }}$ size is bigger than a threshold, all infeasible points are discarded. The effect of these procedures in situations as the one generated by region I, as described in the previous section, is shown in Fig. 2.

To explain this situation, consider one cluster belonging to $\mathrm{P}_{\text {off }}$ as shown in Fig. 2. In this cluster there are several infeasible individuals and just one feasible. When the choice of individuals to be eliminated is made randomly, it is more probable that one infeasible individual will survive despite of the feasible one. Nevertheless, when the modifications aforementioned are used, the feasible individual will remain in $\mathrm{P}_{\text {off }}$ and the infeasible ones will be discarded. Hence, the algorithm with this modified elitism is more likely to converge to the desired feasible PO front than one with the standard elitist technique. This modified P\&M will be denoted by M-P\&M. Without these procedures the nondominated set can partially converge toward an infeasible region due to reinserting many infeasible individuals of $\mathrm{P}_{\text {off }}$ in $\mathrm{P}_{\text {on }}$. It happens because the infeasible individuals can guide the search to the infeasible region. Thus, in the elitism process the constraints are not always viewed as objectives. Eliminating individuals by distance criterion is done to preserve genetic diversity avoiding premature convergence. Distance measuring is done without considering the constraints, because diversity is needed only in the original objectives. When using the classical approach (penalty functions) these procedures are not needed because just feasible individuals are placed in $\mathrm{P}_{\text {off }}$. 


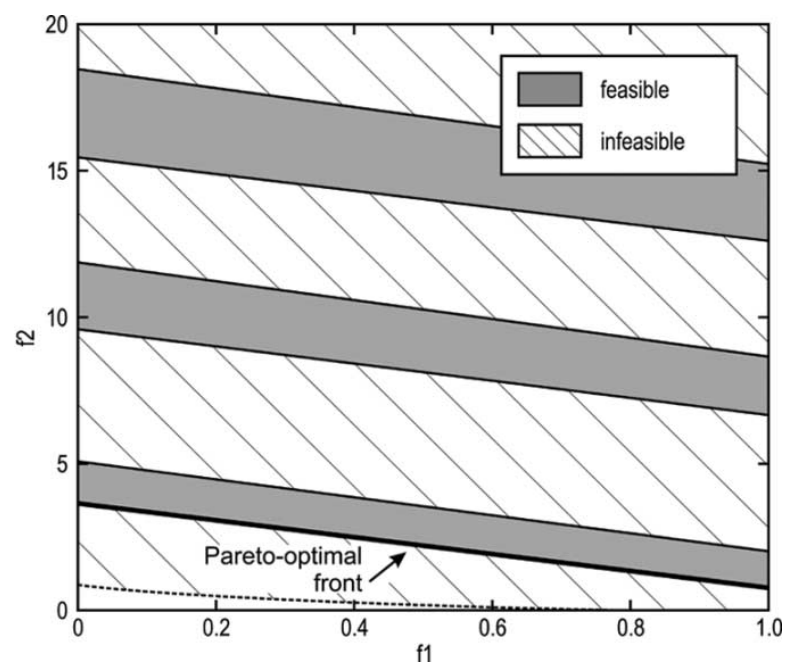

Fig. 3. Constrained test problem CPT6.

\section{Analytical Test Functions}

Four test functions, that were designed with special features to difficult the search [5], were used to compare the classical approach (CA) as formulated in (3) and the new approach (NA), as in (4). To contrast the classical and new approaches with and without elitist techniques, two metrics were used: GD, which is a measure of the distance between the desired and found front and the number of unconstrained individuals $\left(\mathrm{N}_{\text {off }}\right)$ in the $\mathrm{P}_{\text {off }}$ at the end of the simulation [4]. The first test function, TBU, has the following properties: 1) the feasible region is nonconvex and 2) some feasible PO solutions lie on boundaries between the feasible and infeasible regions. This problem is defined as

$$
\begin{aligned}
& \text { minimize } \\
& \bar{f}=\left\{\begin{array}{l}
f_{1}(\bar{x}) \\
f_{2}(\bar{x})
\end{array}\right\}=\left\{\begin{array}{c}
4 x_{1}^{2}+4 x_{2}^{2} \\
\left(x_{1}-5\right)^{2}+\left(x_{2}-5\right)^{2}
\end{array}\right\} \\
& \text { subjected to } \\
& \bar{g}=\left\{\begin{array}{l}
g_{1}(\bar{x}) \\
g_{2}(\bar{x})
\end{array}\right\}=\left\{\begin{array}{c}
\left(x_{1}-1\right)^{2}+x_{2}^{2}-25 \\
-\left(x_{1}-8\right)^{2}-\left(x_{2}+3\right)^{2}+7.7
\end{array}\right\} \\
& \leq\left\{\begin{array}{l}
0 \\
0
\end{array}\right\}
\end{aligned}
$$

where $-15 \leq x_{i} \leq 30, i=1,2$.

This problem was simulated with population size and generation number equal to 80 and 100 , respectively.

The second test function, CPT6, has infeasible holes of different widths toward the PO front (see Fig. 3). This problem is mathematically defined as

minimize

$$
\bar{f}=\left\{\begin{array}{l}
f_{1}(\bar{x}) \\
f_{2}(\bar{x})
\end{array}\right\}=\left\{\begin{array}{c}
x_{1} \\
c(\bar{x})\left[1-\frac{f_{1}(\bar{x})}{c(\bar{x})}\right]
\end{array}\right\}
$$

subjected to

$$
\begin{aligned}
g(\bar{x}) & =\cos (\theta)\left[f_{2}(\bar{x})-e\right]-\sin (\theta) f_{1}(\bar{x}) \\
& \geq a\left|\sin \left\{b \pi\left[\sin (\theta)\left(f_{2}(\bar{x})-e\right)+\cos (\theta) f_{1}(\bar{x})\right]^{c}\right\}\right|^{d}
\end{aligned}
$$

where $c(\bar{x})=41+\sum_{i=2}^{5}\left[x_{i}^{2}-10 \cos \left(2 \pi x_{i}\right)\right]$

$$
\begin{aligned}
& \theta=.01 \pi, a=40, b=0.5, c=1, d=2, e=-2, \\
& 0 \leq x_{1} \leq 1, \quad-5 \leq x_{i} \leq 5, \quad i=2,3,4,5 .
\end{aligned}
$$

This problem was simulated with population size and generation number equal to 40 and 400 , respectively. The main property of the

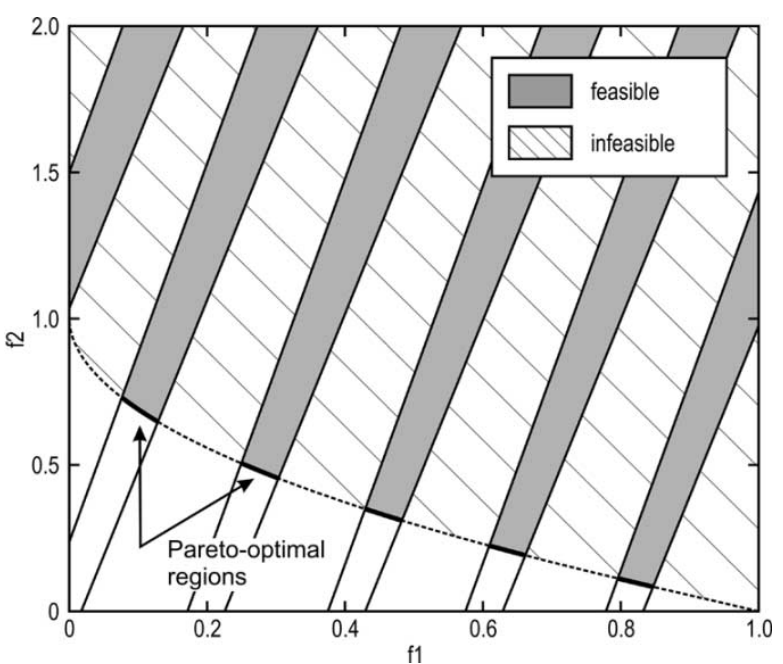

Fig. 4. Constrained test problem CPT7.

third test function, CPT7, is its disconnected PO front (see Fig. 4). It is mathematically stated as CPT6, with: $\theta=-0.05 \pi, \mathrm{a}=40$, $\mathrm{b}=5, \mathrm{c}=1, \mathrm{~d}=6$, and $\mathrm{e}=0$.

The fourth function, OSY, is stated as

$$
\begin{aligned}
\underset{f}{\operatorname{minimize}} & =\left\{\begin{array}{l}
f_{1}(\bar{x}) \\
f_{2}(\bar{x})
\end{array}\right\} \\
& =\left\{\begin{array}{c}
\left.-\left[\begin{array}{c}
25\left(x_{1}-2\right)^{2}+\left(x_{2}-2\right)^{2}+\cdots \\
\left(x_{3}-1\right)^{2}+\left(x_{4}-4\right)^{2}+\left(x_{5}-1\right)^{2}
\end{array}\right]\right\} \\
x_{1}^{2}+x_{2}^{2}+x_{3}^{2}+x_{4}^{2}+x_{5}^{2}+x_{6}^{2}
\end{array}\right\}
\end{aligned}
$$

subjected to

$$
\bar{g}=\left\{\begin{array}{l}
g_{1}(\bar{x}) \\
g_{2}(\bar{x}) \\
g_{3}(\bar{x}) \\
g_{4}(\bar{x}) \\
g_{5}(\bar{x}) \\
g_{6}(\bar{x})
\end{array}\right\}\left\{\begin{array}{c}
x_{1}+x_{2}-2 \\
6-x_{1}-x_{2} \\
2-x_{2}+x_{1} \\
2-x_{1}+3 x_{2} \\
4-\left(x_{3}-3\right)^{2}-x_{4} \\
\left(x_{5}-3\right)^{2}+x_{6}-4
\end{array}\right\} \geq\left\{\begin{array}{l}
0 \\
0 \\
0 \\
0 \\
0 \\
0
\end{array}\right\}
$$

where: $0 \leq x_{1}, x_{2}, x_{6} \leq 10 ; 1 \leq x_{3}, x_{5} \leq 5 ; 0 \leq x_{4} \leq 6$.

The NPGA was executed ten times for each test problem. The arithmetic mean of these results using the metrics discussed above are shown in Table I, where NA denotes the new approach of handling constraints as objectives and CA the classical penalty technique.

The results in Table I show that the approach of treating the constraints as objectives outperform the classical approach of using penalty function. It can be seen comparing both metrics to each test function. The new approach generated individuals closer to the desired PO front than the classical one, see the GD for both approaches. Moreover, the number of points in the nondominated front was bigger $\left(\mathrm{N}_{\text {off }}\right)$ when the new approach was used, showing for these problems effectiveness of this new procedure. For the second and third test problems, CPT6 and CPT7, an acceptable front was only found by using the new approach. The front found for the CPT7 function is shown in Fig. 5. It is important to remember that just feasible individuals were saved, being all infeasible ones discarded at the end of simulation.

The front shown in Fig. 5 when using the classical penalty function is completely dominated by the one found using the new approach. As the constraint is extremely nonlinear, the feasible front is disconnected, being each part of the feasible front separated by 
TABLE I

RESULTS OF ANALYTICAL PROBLEMS

\begin{tabular}{ccccc}
\hline Test functions & \multicolumn{2}{c}{$\mathrm{NA}$} & \multicolumn{2}{c}{$\mathrm{CA}$} \\
\cline { 2 - 5 } & $\mathrm{GD}$ & $\mathrm{N}_{\text {off }}$ & $\mathrm{GD}$ & $\mathrm{N}_{\text {off }}$ \\
\hline TBU & 0.016 & 27.7 & 0.017 & 22.4 \\
\hline CPT6 & 0.0037 & 30 & .175 & 8.4 \\
\hline CPT7 & 0.0025 & 12.5 & 0.0615 & 2.1 \\
\hline OSY & 0.32 & 57 & 0.99 & 30.6 \\
\hline
\end{tabular}

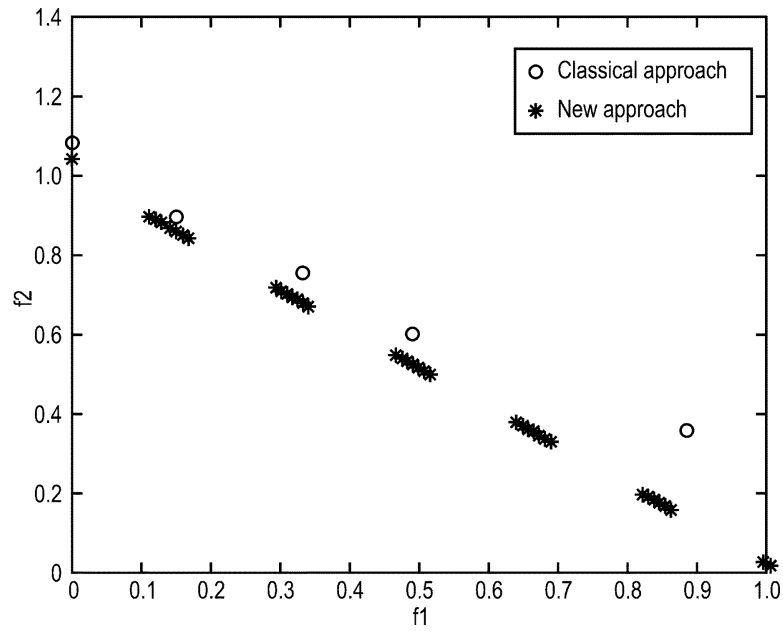

Fig. 5. Nondominated sets of CPT7 problem.

an infeasible one. This case confirms that is good keeping infeasible individuals that are near the PO front in the on-line population as is done in the new approach. This does not happen when using penalty function approach, causing convergence to an incorrect nondominated front

\section{CONSTRAined MultiobJECTIVE OptimizATION IN ELECTROMAGNETICS}

The TEAM Benchmark Problem 22 was chosen to demonstrate the performance of the new approach in electromagnetics optimization problems. The aim of this problem is to optimize the Super-Conducting Energy Storage configuration with respect to two objectives and one constraint, to ensure minimal stray field $\left(f_{1}\right), 180 \mathrm{MJ}$ of stored energy $\left(\mathrm{f}_{2}\right)$ and that physical quench condition is met $(\mathrm{g})$. The complete description of the problem is presented in [6], so it is omitted here. This problem was specified with three continuous variables, keeping the other fixed. It was solved using NPGA with both new and classical approaches to handle the physical quench condition. In both cases the modified and standard Parks \& Miller elitisms were used and the population size and generation number were fixed to 30 . The analysis of this problem was realized using a finite element code using triangular elements of first order. The results are presented in Fig. 6. The front found

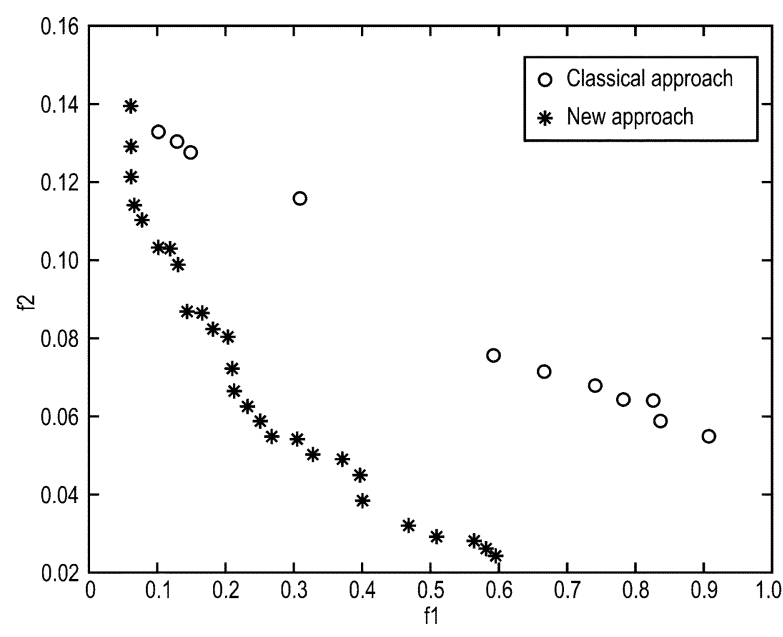

Fig. 6. Nondominated sets of TEAM22 problem.

using the classical approach was completely dominated by one found using the new approach. The simulation for this problem spent $3 \mathrm{~h}$ and 13 min using the NA and $3 \mathrm{~h}$ and 5 min considering the CA. Note that this difference is not important to numerical problems as TEAM22.

\section{CONCLUSION}

The results obtained by handling constraints as objectives when solving both test and TEAM'22 problems demonstrate that this approach outperform the classical one. As discussed earlier, the standard Parks \& Miller elitism has been modified to consider the constraints transformed into objectives. The proposed modifications were important to avoid the convergence of NPGA toward infeasible regions and to represent better the nondominated front. These results pointed out the effectiveness of this new approach in multiobjective optimization problem. Moreover, the computational effort is almost the same on both situation.

\section{REFERENCES}

[1] J. Horn, N. Nafpliots, and E. Goldberg, "A niched pareto genetic algorithm for multiobjective optimization," in Proc. 1st IEEE Conf. Evolutionary Computation/IEEE World Congr. Computational Intelligence, vol. 1, June 1994, pp. 82-87.

[2] D. A. G. Vieira, R. L. S. Adriano, L. Kraähenbül, and J. A. Vasconcelos, "Handling constraints as objectives in a multiobjective genetic based algorithm," J. Microwaves Optoelectron., vol. 2, no. 6, pp. 50-58, Dec. 2002.

[3] G. T. Parks and G. T. Miller, "Selective breeding in a multiobjective genetic algorithm," in Parallel Problem Solving From Nature PPSN V. Berlin, Germany: Springer-Verlag, 1998, pp. 250-259.

[4] D. A. Van Veldhuizen and G. B. Lamont, "On measuring multiobjective evolutionary algorithm performance," in Proc. 2000 Congr. Evolutionary Computation, vol. 1, July 2000, pp. 204-211.

[5] K. Deb, A. Pratap, and T. Meyarivan, "Constrained test problems for multi-objective evolutionary optimization," in First International Conference on Evolutionary Multi-Criterion Optimization, ser. Lecture Notes in Computer Science. Berlin, Germany: Springer-Verlag, 2001, vol. 1993, pp. 284-298.

[6] Tech. Univ. Graz, Graz, Austria. TEAM Optimization Benchmark Problem 22. [Online]. Available: http://www-igte.tu-graz.ac.at/team/ team3dis.htm 